\title{
Cutaneous Vasculopathy Associated with Levamisole-Adulterated Cocaine
}

\author{
Huy Tran, MD, PhD; Debbie Tan, MD; and Thomas P. Marnejon, DO
}

\begin{abstract}
We report a case of cutaneous vasculopathy associated with the use of levamisole-adulterated cocaine. This recently described clinical entity is characterized by a purpuric rash with a predilection for the ears, leukopenia, and anti-neutrophilic cytoplasmic antibody (ANCA) positivity. It is estimated that more than $70 \%$ of the current United States cocaine supply is contaminated with levamisole. Levamisole is a widely available, inexpensive, white powder used as a "cutting agent" in cocaine to expand volume and increase profits. It may also increase the euphoric and stimulatory effects of cocaine by increasing brain dopamine levels and producing amphetamine-like metabolites. Our patient exhibited a characteristic rash with involvement of the ears, leukopenia, and cocaine metabolites were detected in serum and urine. The presence of levamisole was confirmed in the urine utilizing gas chromatography-mass spectrometry. ANCA positivity was also present. Punch biopsy of the skin demonstrated vascular thrombosis and necrosis without true vasculitis. We review the literature for reported cases of cocainelevamisole cutaneous vasculopathy syndrome, highlight the salient immunologic abnormalities, and contrast the features of this entity with idiopathic systemic vasculitis.
\end{abstract}

Keywords: Anti-neutrophilic cytoplasmic antibody; Cocaine; Cutaneous vasculopathy; Levamisole

Corresponding Author:

Thomas Marnejon, DO

Department of Medicine

St. Elizabeth Health Center

1044 Belmont Avenue

Youngstown, $\mathrm{OH} 4450 \mathrm{I}$

Tel: (330) 480-3344

Fax: (330) 480-3777

Email: thomas_marnejon@hmis.org

Received: March 6, 2012

Revised: May 10, 2012

Accepted: May 16, 2012

doi: $10.3121 / \mathrm{cmr} .2012 .1085$
A with a rash on his ears and legs of one week duration. The lesions on his ears were described as painful. He also had arthralgias of his hands, but denied fever, chills, sore throat, and cough. He also denied epistaxis, hemoptysis, hematemesis, melena, hematuria, and Raynaud's phenomenon. History was significant for chronic back pain, chronic hepatitis $\mathrm{C}$ infection, and intermittent cocaine use. There were no known drug allergies; his only medication was over the counter ibuprofen for pain.

On physical examination, the patient was afebrile. Examination of the skin revealed a violaceous, non-blanching rash in a retiform pattern with areas of necrosis located on the helix and earlobes bilaterally (figure 1A) and both knees (figure 1B). There were no mucosal lesions, lymphadenopathy, or hepatosplenomegaly. Laboratory testing showed a white blood cell (WBC) count of 3200 per $\mathrm{mm}^{3}$ (normal range of $4500-11,500 \mathrm{per} \mathrm{mm}^{3}$ ). The platelet count was 148,000 per $\mathrm{mm}^{3}$. Hemoglobin, coagulation studies, urinalysis, basic metabolic panel, renal and hepatic functions were normal. Erythrocyte sedimentation rate and C-reactive protein were normal. Toxicology revealed a positive cocaine metabolite, benzoylecgonine, in urine utilizing gas chromatography-mass spectrometry (GC-MS) (Arup Laboratories, Salt Lake City, UT). Levamisole was also detected in the urine via GC-MS (Toxicology Laboratory, Colorado Department of Public Health and Environment). 

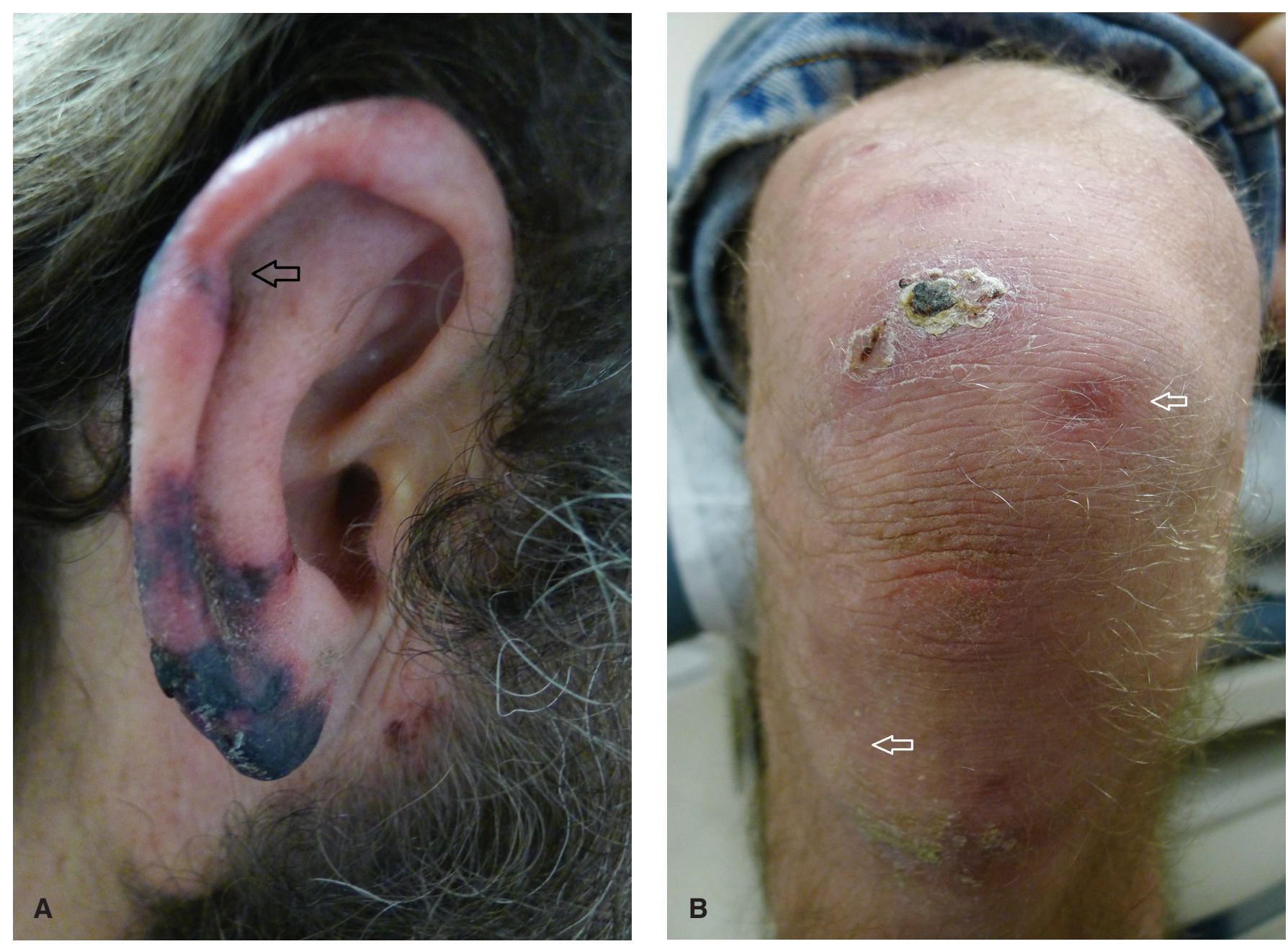

Figure 1. Tender violaceous, non-blanching rash with areas of necrosis located on $(A)$ the right earlobe and $(B)$ right knee. There are scars on both sites from previous exposure to cocaine (open arrows).

Approximately one year before the current presentation, the patient had been admitted for a similar rash that was present on his ears, elbows, legs, and trunk. At that time the rash was described as macular, violaceous, and non-blanching, with areas of central necrosis. Tests for rheumatoid factor, cryoglobulin, and anti-nuclear antibody (ANA) were negative. Complement $\mathrm{C} 3$ and $\mathrm{C} 4$ were within normal range $(65 \mathrm{mg} / \mathrm{dL}$ and $14 \mathrm{mg} / \mathrm{dL}$, respectively). Enzyme-linked immunosorbent assay (ELISA) for anti-proteinase 3 anti-neutrophilic cytoplasmic antibodies (PR3-ANCA) was positive at $43 \mathrm{AU} /$ $\mathrm{ml}$ (normal 0-19 AU/ml). ELISA for anti-myeloperoxidase (MPO-ANCA) was also mildly positive at $22 \mathrm{AU} / \mathrm{ml}$ (normal $0-19 \mathrm{AU} / \mathrm{ml}$ ), which became negative on repeat testing, as the skin lesions improved. Immunoglobulin $M$ (IgM) anti-cardiolipin antibody was $35 \mathrm{MPL} \mathrm{U} / \mathrm{ml}$ (normal 0-12 MPL U/ml). Lupus-like anticoagulant was weakly positive. Testing for human immunodeficiency virus, parvovirus, cytomegalovirus, and Ebstein-Barr virus were all negative. The cocaine metabolite, benzoylecgonine, was detected in the urine. A punch biopsy of the lesion over the patient's elbow demonstrated microthrombi with overlying epidermal necrosis (figures $2 \mathrm{~A}$ and $2 \mathrm{~B}$ ). The patient was treated with high dose prednisone $(1 \mathrm{mg} / \mathrm{kg} /$ day $)$ for 2 months that was tapered to a dose of $15 \mathrm{mg} /$ day. The rash on his trunk and extremities improved, while the rash on the earlobes completely resolved. Prednisone was discontinued 5 months before this present emergency room encounter. The patient reported cessation of cocaine use during that same time period.

\section{Discussion}

The differential diagnosis of retiform purpura, which is characterized by stellate or branching purpuric lesions, is extensive and includes small and medium-sized vessel vasculitis, infectious and embolic phenomenon, warfarin skin necrosis, disseminated intravascular coagulation, cryoglobulinemia, and anti-phospholipid antibody syndrome. In users of cocaine contaminated with levamisole, a cutaneous vasculopathy syndrome has recently emerged and is characterized by a distinctive purpuric rash with a predilection for the ears, ANCA positivity, and leukopenia. ${ }^{1-3}$

Levamisole, an antihelminthic with immunomodulatory properties, was previously used for the treatment of autoimmune disorders, pediatric nephritic syndrome, and cancer. It was withdrawn from the market in 2000 due to its adverse side effect profile, specifically agranulocytosis. 

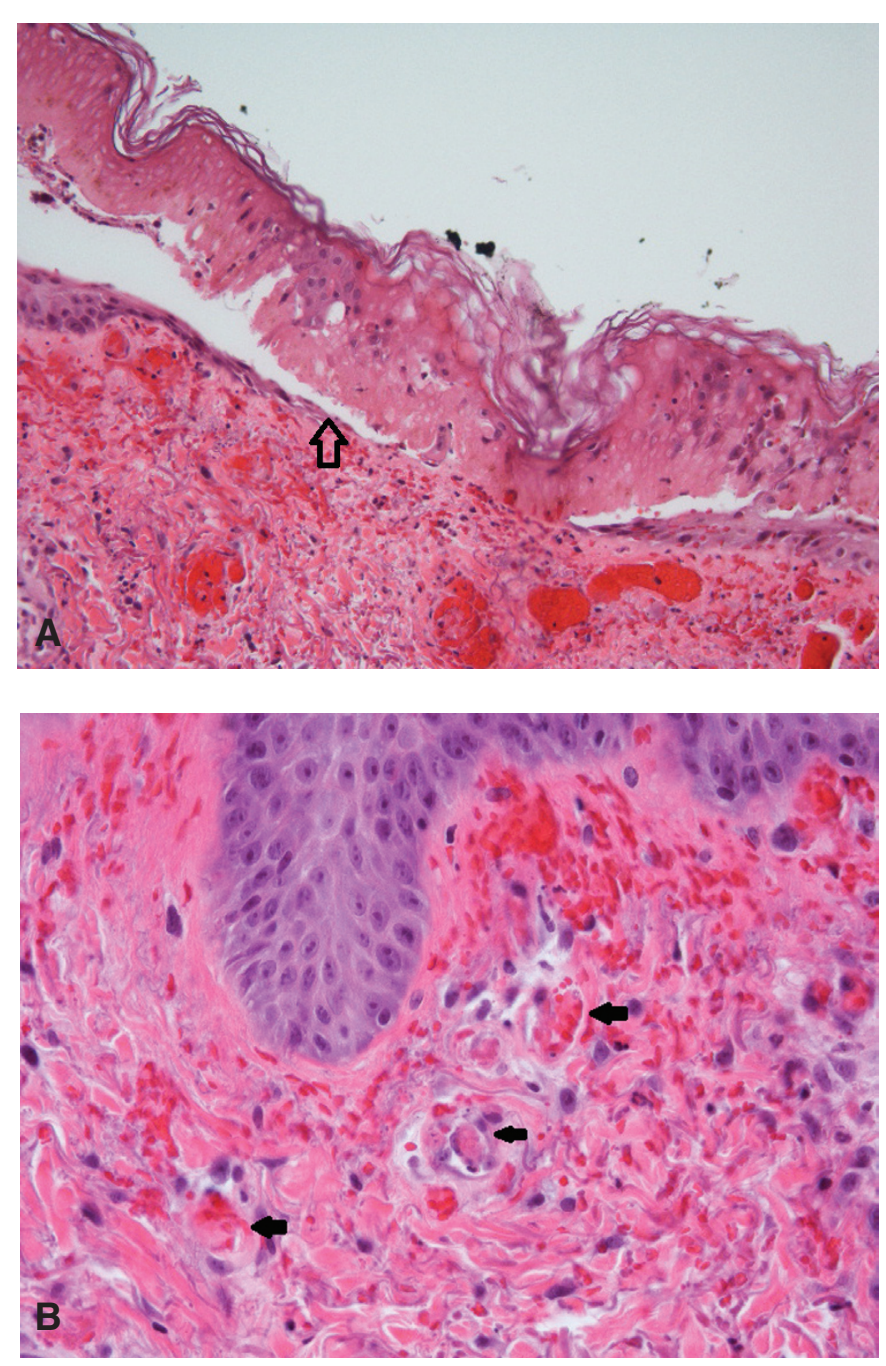

Figure 2. Punch biopsy specimen of the skin which shows $(A)$ epidermal necrosis (open arrow) with (B) intravascular fibrin thrombi of superficial and mid-dermal vessels (black arrows). Hematoxylin-eosin stain, magnification x40.

Levamisole is now found as an adulterant in as much as $70 \%$ to $88 \%$ of cocaine in the United States. ${ }^{4,5}$ Levamisole is a widely available, inexpensive, white powder that is used as a "cutting agent" in cocaine to expand volume and increase profits. Levamisole may also increase the euphoric and stimulatory effects of cocaine by increasing brain dopamine levels and producing amphetamine-like metabolites. ${ }^{6}$ Cutaneous manifestations associated with levamisole use are varied and include nonspecific eruptions, lichenoid eruptions, fixed drug rash, and cutaneous vasculitis. ${ }^{7}$ Lesions may appear suddenly and enlarge rapidly. Purpuric papules, plaques, hemorrhagic bullae, and even midline destruction have also been reported..$^{7-9}$ In the cocaine-levamisole cutaneous vasculopathy syndrome, lesions frequently have a distinctive morphology; they tend to be stellate with a bright erythematous border and necrotic appearing center. ${ }^{1,10}$ One of the most unique features of this syndrome is that the rash has a predilection for the ears, which has been documented in many case reports and case series. ${ }^{2,3,8,11,12}$ It is interesting to speculate on the reason why the ears are preferentially involved. It may be due to the fact that the lower temperature of the ear may facilitate the deposition of immune complexes. ${ }^{7}$ The histopathologic findings of cocaine-levamisole cutaneous vasculopathy syndrome range from leukocytoclastic and thrombotic vasculitis to vascular occlusive disease without true vasculitis. ${ }^{1}$ Biopsy features may show necrosis of small and medium-sized vessels of the dermis and subcutis or an occlusive vasculopathy with intravascular fibrin thrombi and epidermal necrosis without vasculitis. ${ }^{2,12}$

Detection of levamisole in both serum and urine must be performed using GC-MS or liquid chromatography-tandem mass spectrometry (LC-MS), as it is not detectable by routine toxicologic testing. The half-life of levamisole is approximately 5.6 hours, and limited amounts are excreted in the urine, which can make detection difficult. ${ }^{13}$ In a recent review of 16 cases of cutaneous vasculitis syndrome secondary to levamisole-adulterated cocaine, Gross et $\mathrm{al}^{1}$ noted that only $12.5 \%$ of patients tested positive for levamisole. Based upon the characteristic clinical picture and the widespread adulteration of cocaine with levamisole, some authors believe that a positive test for levamisole is not required to make the diagnosis of this syndrome. ${ }^{1,14}$

Cocaine-levamisole cutaneous vasculopathy syndrome has unique immunologic abnormalities, the most important of which is ANCA positivity. ${ }^{1,2,7,8,14-16}$ This can present a significant diagnostic challenge and renders it difficult to distinguish from true idiopathic systemic vasculitis. In cocaine-levamisole vasculopathy patients, high titer ANCAs against multiple antigens, higher incidence of antiphospholipid antibodies, and low titerANA, are characteristic. ${ }^{14}$ Specifically, these patients may have high titer perinuclear-ANCA (p-ANCA) positivity alone, ${ }^{2,8}$ or characteristically, both p-ANCA and cytoplasmic-ANCA (c-ANCA), with anti-MPO and anti-PR3 positivity. ${ }^{1,7,14,16}$ In systemic vasculitis, reactivity to anti-PR3 alone is sensitive and specific to Wegener granulomatosis, whereas reactivity to anti-MPO alone is typically associated with microscopic polyangiitis and Churg-Strauss syndrome. Low titer ANA and antiphospholipid-antibodies have been reported in several cases. ${ }^{7,14,16,17}$ Recently, anti-human neutrophil elastase (HNE), a subset of ANCA, has been reported to distinguish the cocaine-levamisole vasculopathy syndrome from true autoimmune vasculitis. ${ }^{18}$

ANCA-associated vasculitis has been linked to various drugs, such as hydralazine, propylthiouracil, and minocycline. ${ }^{19}$ Immunologic abnormalities found in drug-induced ANCAassociated vasculitis include high titer anti-MPO antibodies or combined positivity of both anti-MPO and anti-PR3, ANA, and anti-cardiolipin antibodies, similar to that observed in cocaine-levamisole cutaneous vasculopathy. ${ }^{19}$ Typically, a prolonged exposure to a causative drug is required before the development of drug-induced autoantibody production. Rongioletti et $\mathrm{al}^{7}$ documented a mean latency of 24 months of 
exposure to levamisole before the development of circulating antibodies. ANCA and anti-phospholipid antibodies induced by levamisole exposure normalize within 2 to 14 months after discontinuation of the drug. ${ }^{7}$

The association of levamisole with leukopenia and agranulocytosis in the treatment of autoimmune disorders and cancer has been well documented. ${ }^{20}$ Similarly, there have been multiple reports of leukopenia and agranulocytosis secondary to the use of cocaine contaminated with levamisole. ${ }^{21-24}$ Gross et al ${ }^{1}$ reported that 10 out of 16 patients $(63 \%)$ with this syndrome had either leukopenia with WBC count less than 4000 per $\mathrm{mm}^{3}$ or neutropenia with ANC less than 1500 per $\mathrm{mm}^{3}$. Of note, leukopenia was present in our patient.

Presently, it is unclear why this syndrome develops in certain cocaine users. It has been postulated that the presence of 6-phenyl-2,3-dihydroimidazo(2,1b)-thiazole, one of the three breakdown products of levamisole, with lymphocytestimulating effects, could play a role in pathogenesis. ${ }^{14,25} \mathrm{An}$ association between levamisole-induced agranulocytosis and HLA-B27 positivity has also been documented. ${ }^{26}$

Currently, there is no consensus on the treatment of cocainelevamisole cutaneous vasculopathy syndrome. It is unclear whether steroids offer any benefit, and their use must be balanced against side effects. ${ }^{1}$ The role of other immunosuppressants is also controversial, especially when patients present with leukopenia or agranulocytosis. ${ }^{16}$ As with other drug-induced vasculitides, resolution can be hastened by withdrawing the offending drug. Recurrent skin lesions do occur when patients are re-challenged with levamisoleadulterated cocaine. ${ }^{17}$ Recurrent lesions on re-challenge was noted in our patient; purpuric lesions on the earlobes and knees recurred after using cocaine again (figure 1).

\section{Summary}

We present a case of cutaneous vasculopathy associated with levamisole-adulterated cocaine as manifested by a stellate purpuric rash with involvement of the ears and leukopenia. Levamisole was detected in the urine, and ANCA positivity was present. Pathologic findings, including microthrombi and epidermal necrosis, were also consistent with the clinical diagnosis. Cutaneous vasculopathy associated with levamisole-adulterated cocaine, a recently described clinical entity, is characterized by a purpuric rash in a retiform pattern with predilection for the ears, leukopenia, and ANCA positivity. This syndrome may be difficult to distinguish from a true systemic vasculitis. Characteristic serologic abnormalities of cocaine-levamisole vasculopathy include high titer p-ANCA alone or both p-ANCA and c-ANCA positivity with anti-MPO and anti-PR3 antibodies. Low titer ANA, antiphospholipid antibodies, and leukopenia are frequently present. The absence of end organ involvement with isolated skin manifestations, along with a typical serologic profile, differentiates cocaine-levamisole cutaneous vasculopathy from idiopathic systemic vasculitis.
Given the frequency of cocaine use in the United States and the high rate of levamisole-adulteration, the diagnosis of cocaine-levamisole cutaneous vasculopathy syndrome should be suspected in any patient presenting with a purpuric rash, especially when the ear is involved, leukopenia is present, or there is a positive ANCA test.

\section{Acknowledgements}

We wish to thank David Gemmel, $\mathrm{PhD}$ for critical review and support of the submitted manuscript, and the Toxicology Laboratory, Colorado Department of Public Health and Environment.

\section{References}

1. Gross RL, Brucker J, Bahce-Altuntas A, Abadi MA, Lipoff J, Kotlyar D, Barland P, Putterman C. A novel cutaneous vasculitis syndrome induced by levamisolecontaminated cocaine. Clin Rheumatol 2011; 30:1385-1392.

2. Jenkins J, Babu K, Hsu-Hung E, Robinson-Bostom L, Kroumpouzos G. ANCA-positive necrotizing vasculitis and thrombotic vasculopathy induced by levamisoleadulterated cocaine: a distinctive clinicopathologic presentation. J Am Acad Dermatol 2011;65:e14-e16.

3. Menni S, Pistritto G, Gianotti R, Ghio L, Edefonti A. Ear lobe bilateral necrosis by levamisole-induced occlusive vasculitis in a pediatric patient. Pediatr Dermatol 1997;14:477-479.

4. Buchanan JA, Heard K, Burbach C, Wilson ML, Dart R. Prevalence of levamisole in urine toxicology screens positive for cocaine in an inner-city hospital. JAMA 2011;305:1657-1658.

5. Lynch KL, Dominy SS, Graf J, Kral AH. Detection of levamisole exposure in cocaine users by liquid chromatography-tandem mass spectrometry. J Anal Toxicol 2011;35:176-178.

6. Chang A, Osterloh J, Thomas J. Levamisole: a dangerous new cocaine adulterant. Clin Pharmacol Ther 2010;88:408-411.

7. Rongioletti F, Ghio L, Ginevri F, Bleidl D, Rinaldi S, Edefonti A, Gambini C, Rizzoni G, Rebora A. Purpura of the ears: a distinctive vasculopathy with circulating autoantibodies complicating long-term treatment with levamisole in children. Br J Dermatol 1999; 140:948-951.

8. Farmer RW, Malhotra PS, Mays MP, Egger ME, Smith JW, Jortani SA, Spiller H, Bosse GM, Callen JP, Franklin GA. Necrotizing peripheral vasculitis/ vasculopathy following the use of cocaine laced with levamisole. J Burn Care Res 2012;33:e6-e11.

9. Ching JA, Smith DJ Jr. Levamisole-induced necrosis of skin, soft tissue, and bone: case report and review of literature. J Burn Care Res 2012;33:e1-e5.

10. Geller L, Whang TB, Mercer SE, Phelps R. Retiform purpura: a new stigmata of illicit drug use? Dermatol Online J 2011;17:7. 
11. Buchanan JA, Vogel JA, Eberhardt AM. Levamisoleinduced occlusive necrotizing vasculitis of the ears after use of cocaine contaminated with levamisole. J Med Toxicol 2011;7:83-84.

12. Bradford M, Rosenberg B, Moreno J, Dumyati G. Bilateral necrosis of earlobes and cheeks: another complication of cocaine contaminated with levamisole. Ann Intern Med 2010;152:758-759.

13. Kouassi E, Caillé G, Léry L, Larivière L, Vézina M. Novel assay and pharmacokinetics of levamisole and p-hydroxylevamisole in human plasma and urine. Biopharm Drug Dispos 1986;7:71-89.

14. Ullrich K, Koval R, Koval E, Bapoje S, Hirsh JM. Five consecutive cases of a cutaneous vasculopathy in users of levamisole-adulterated cocaine. J Clin Rheumatol 2011;17:193-196.

15. Friedman DR, Wolfsthal SD. Cocaine-induced pseudovasculitis. Mayo Clin Proc 2005;80:671-673.

16. McGrath MM, Isakova T, Rennke HG, Mottola AM, Laliberte KA, Niles JL. Contaminated cocaine and antineutrophil cytoplasmic antibody-associated disease. Clin J Am Soc Nephrol 2011;6:2799-2805.

17. Walsh NM, Green PJ, Burlingame RW, Pasternak S, Hanly JG. Cocaine-related retiform purpura: evidence to incriminate the adulterant, levamisole. J Cutan Pathol 2010;37:1212-1219.

18. Wiesner O, Russell KA, Lee AS, Jenne DE, Trimarchi M, Gregorini G, Specks U. Antineutrophil cytoplasmic antibodies reacting with human neutrophil elastase as a diagnostic marker for cocaine-induced midline destructive lesions but not autoimmune vasculitis. Arthritis Rheum 2004;50:2954-2965.

19. Gao Y, Zhao MH. Review article: Drug-induced antineutrophil cytoplasmic antibody-associated vasculitis. Nephrology (Carlton). 2009;14:33-41.

20. Thompson JS, Herbick JM, Klassen LW, Severson CD, Overlin VL, Blaschke JW, Silverman MA, Vogel CL. Studies on levamisole--induced agranulocytosis. Blood 1980;56:388-396.

21. Buchanan JA, Oyer RJ, Patel NR, Jacquet GA, Bornikova L, Thienelt C, Shriver DA, Shockley LW, Wilson ML, Hurlbut KM, Lavonas EJ. A confirmed case of agranulocytosis after use of cocaine contaminated with levamisole. J Med Toxicol 2010;6:160-164.

22. Mouzakis J, Somboonwit C, Lakshmi S, Rumbak M, Sinnott J, Cherpelis B, Keshishian J. Levamisole induced necrosis of the skin and neutropenia following intranasal cocaine use: a newly recognized syndrome. J Drugs Dermatol 2011;10:1204-1207.

23. Centers for Disease Control and Prevention (CDC). Agranulocytosis associated with cocaine use - four States, March 2008-November 2009. Morb Mortal Wkly Rep 2009;58:1381-1385.

24. Zhu NY, Legatt DF, Turner AR. Agranulocytosis after consumption of cocaine adulterated with levamisole. Ann Intern Med 2009;150:287-289.
25. Hanson KA, Heidrick ML. Immunomodulatory action of levamisole--II. Enhancement of concanavalin A response by levamisole is associated with an oxidation degradation product of levamisole formed during lymphocyte culture. Int J Immunopharmacol 1991;13:669-676.

26. Czuchlewski DR, Brackney M, Ewers C, Manna J, Fekrazad MH, Martinez A, Nolte KB, Hjelle B, Rabinowitz I, Curtis BR, McFarland JG, Baumbach J, Foucar K. Clinicopathologic features of agranulocytosis in the setting of levamisole-tainted cocaine. Am J Clin Pathol 2010;133:466-472.

\section{Author Affiliations}

Huy Tran, MD, PhD*; Debbie Tan, MD*; and Thomas $P$. Marnejon, $D O^{* \dagger}$

*Internal Medicine Residency Program, Department of Medicine, St. Elizabeth Health Center, Youngstown, Ohio, USA

tDepartment of Medicine, Northeast Ohio Medical University, Rootstown, Ohio, USA 